\title{
MACROSCOPIC FUNDAMENTAL DIAGRAMS: SIMULATION FINDINGS FOR THESSALONIKI'S ROAD NETWORK
}

\author{
Iraklis Stamos ${ }^{1}$, Josep Maria Salanova Grau ${ }^{2}$, Evangelos Mitsakis ${ }^{3}$, Socratis Mamarikas ${ }^{4}$ \\ 1,2,3,4 Hellenic Institute of Transport (CERTH/HIT), Greece
}

Received 26 March 2015; accepted 9 July 2015

\begin{abstract}
Within the framework of this paper, the existence of a Macroscopic Fundamental Diagram (MFD) for the city of Thessaloniki is discussed, obtained through simulation. Initial findings show that there is a well-defined MFD, which is a property of a network, as it is not influenced by changes in travel demand. The MFD can be used as tool for managing traffic at real-time and for improving accessibility in an area, through pricing strategies and entrance points control based on the accumulation of vehicles.
\end{abstract}

Keywords: macroscopic fundamental diagram, traffic simulation, traffic flow, traffic density.

\section{Introduction}

Conventionally, traffic is characterized by three key variables: the flow (number of vehicles per time unit), the speed and the density (or occupancy) (number of vehicles per length unit). The relationships between these variables initially identified through empirical observations on continuous (noninterrupted) traffic flow streams, to specific sections of motorways (Greenshields, 1934; Greenberg, 1959) are called Fundamental Diagrams (FD) of traffic for a road section. As non-interrupted, is defined the traffic flow where a vehicle does not have to stop or decelerate due to any cause other than the traffic stream, such as the existence of traffic lights or signs at intersections. When the variables of flow, speed and density are estimated as average values at a road network-level, then these are defined as its macroscopic characteristics and the relationships that arise between them are named Macroscopic Fundamental Diagrams (MFD), in contrast to the Fundamental Diagrams (FD) that refer to specific road sections. As it is presented in Fig. 1, the relation between speed and traffic flow has a parabolic form, wherein the average speed decreases as the flow increases up to a maximum flow (the road capacity) under free flow conditions and then both the flow and speed are reduced, so as to describe traffic congestion.

\footnotetext{
${ }^{3}$ Corresponding author: emit@certh.gr
} 

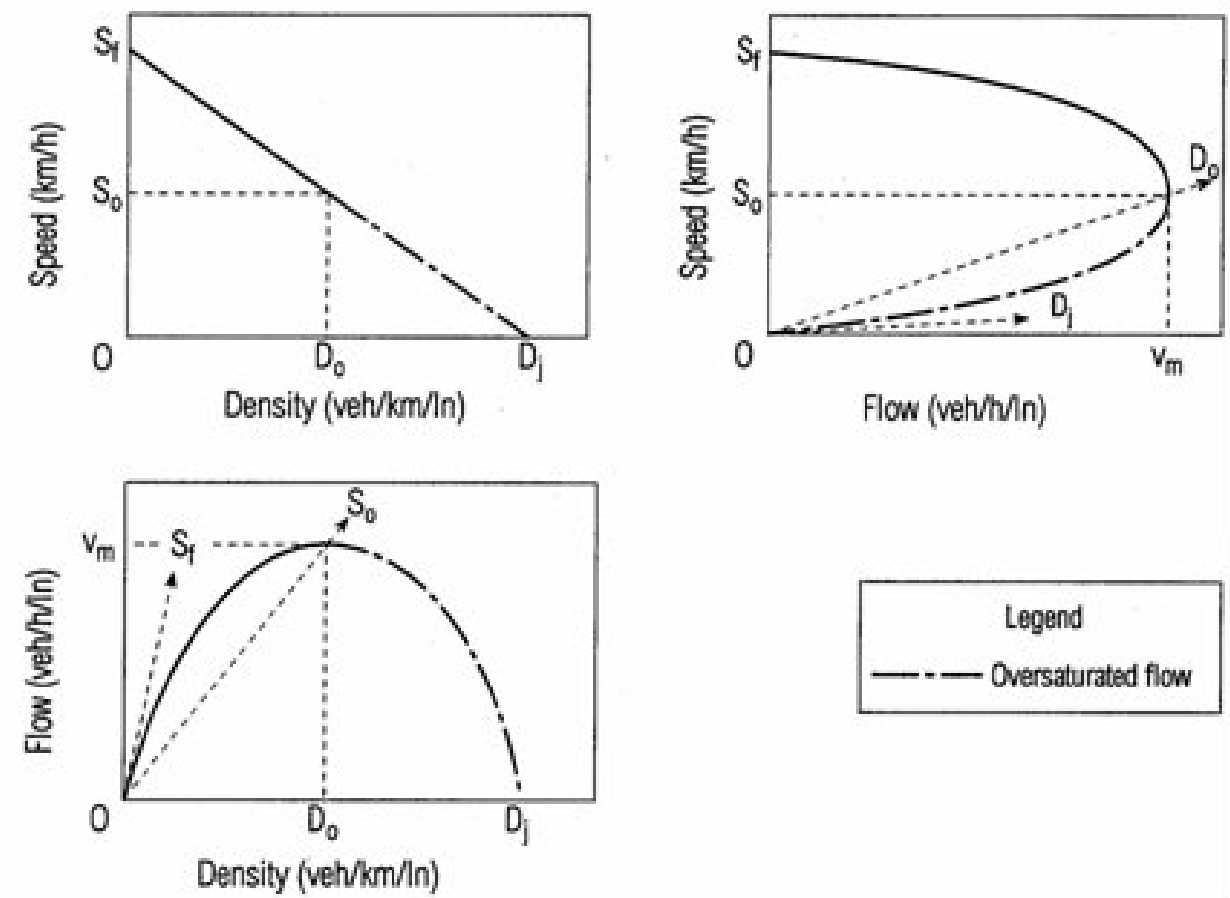

Fig. 1.

Fundamental Diagrams of Speed-Flow-Density

Source: TCSH (2008)

Later, empirical observations showed that similar relationships exist at a network-level, where traffic flow is regularly interrupted by controlled intersections. For instance, Thomson (1967) developed a linear relationship between speed and flow using data that had been collected for several years from streets in central London. Godfrey (1969) found a parabolic relationship between the average travel speed and the vehicle kilometres travelled on the network of central London and showed that the speed is inversely proportional to the density (which is defined as the number of vehicles in the network of central London in a given time). On a more theoretical approach, Wardrop (1968) proposed a general relationship between average speed and flow, which depends on the average width of the road and the average length of the intersection, but which still decreases monotonically. This conclusion had also been made by Zahavi (1972), examining data from urban networks in England and the US, where he found that the average speed is inversely proportional to the flow. But monotonicity had only sense when traffic was low, since it cannot describe situations with very low speeds and flows and therefore cannot be used to describe peak hours of a network. Later, Herman and Prigogine (1979) and Herman and Ardekani (1984) proposed a "2-fluid model” for traffic at cities, in which they assumed that speed distribution is divided into two parts: one 
part corresponding to moving vehicles and another to vehicles that have stopped due to local conditions (congestion, traffic control, accidents etc.).

Williams et al. (1987) and Mahmassani et al. (1987) through a simulation investigated the relationship between the three main variables of traffic flow at a network level, based on the $\ll 2$-fluid $\gg$ model theory. However, the data from these studies were not sufficient to prove the existence of an invariant macroscopic relationship for real urban networks, as their validity was not analysed under conditions of variable demand and the model was not developed to be able to dynamically describe the peak hour of a network. The existence and the uniqueness of a hollow FD for a road with several sections without turns and traffic lights has been proved by Daganzo (2005a, b). Later, he assumed that a well-defined relationship between flow and density can occur if a network is "uniformly congested" in space and the demand is slowly changing with time (Daganzo, 2007).

Geroliminis and Daganzo (2007) reformulated this theory through two axioms: (a) uniformly congested areas (neighbourhoods) present an MFD, correlating the "production" (the product of the mean flow and the network length) with the "accumulation" (the product of density and network length) (b) the completion rate of movements is proportional to production. Several simulations during the peak hour in downtown San Francisco (with very different demand distributions both of space and time) showed that (a) MFDs exist regardless of the demand, (b) the accumulation can be dynamically predicted, (c) the perimeter control systems aiming to improve accessibility operates as expected.
The empirical verification of MFD existence has been made recently through the combination of measurements from 500 detectors at Yokohama city of Japan with a large data sample derived from GPS devices in taxis (Daganzo and Geroliminis, 2008). The analyses and simulations in these reports (Geroliminis and Daganzo, 2007; Daganzo and Geroliminis, 2008) show that MFDs are curves that can be reproduced under homogeneous conditions in urban networks, even if a flow-density FD for individual road sections shows significant dispersion (Daganzo and Geroliminis, 2008). These reports also showed that MFDs are a property of the infrastructure and not of the demand, i.e. the average flow in a network is maximum for the same density value, regardless of the time-varying origindestination tables. Further research has shown that the form and the shape of MFD depend on the demand (Laval, 2010) and the detectors' placement (Buisson and Ladier, 2009), showing a significant dispersion in complex networks (Courbon and Leclerq, 2011). The impact of the spatial distribution of congestion is also an important factor of MFD (Buisson and Ladier, 2009; Ji and Geroliminis, 2011; Knoop et al., 2012; Mazloumian et al., 2010). Various methodologies have been stated, in order to solve the above mentioned issues such as the generalized MFD (Knoop and Hoogendoorn, 2012). The research as regards the existence and the characteristics of MFD, has been expanded at arterial networks (Geroliminis and Sun, 2011a) and motorway networks (Geroliminis and Sun, 2011b; Cassidy et al., 2011).

The aim of this study is to investigate the existence of a flow-density MFD for Thessaloniki's road network, using traffic simulation tools. The remainder of the 
paper is structured as follows: In Section 2, Thessaloniki's road network is presented, the modelling and simulation tools that have been used for extraction of the flow-density MFD are analysed and the input data of these tools are briefly described. Subsequently, the methodology that has been used is provided and the results of the analysis are presented, as well as the flow-density MFD for Thessaloniki's road network. In Section 3, a further analysis of the results is performed and it is examined the way the diagram is affected by the number of road sections used for its extraction in a network, by the time intervals and the duration the results' resolution and by the demand changes. Finally, Section 4 presents the conclusions of the paper.

\section{Thessaloniki's Network}

\subsection{Reference Network}

Thessaloniki is the second largest city in Greece with 1.006 .730 people living in the wider area. The city is located in Northern Greece and covers a total area of $1.455,68 \mathrm{~km}^{2}$, with an average density of 665,2 inhabitants per $\mathrm{km}^{2}$. Due to its geographical location, Thessaloniki has an important social, economic and commercial role, both domestically and in the wider Balkan region (Stamos et al., 2012). The road network of the city includes different categories of roads, while the intersections at the city centre are relatively closely spaced (100m-400m) and controlled by traffic lights (Politis et al., 2012). The roads have one to four (1-4) lanes in each direction and the speed limit on central arterials is $\mathbf{5 0}$ $\mathrm{km} / \mathrm{h}$. Central intersections are controlled by multiphase signalling systems, which alternate, based on the hour and the day (up to 5 daily programs with cycle lengths of 45 , 60, and 90 seconds) (Stamos et al., 2013). The part of Thessaloniki's city centre that is examined in the framework of the present work is approximately $2,86 \mathrm{~km}^{2}$, delineated by the YMCA square, the Sintrivani square, the junction of Langada-Monastiriou, the Dikastiria square and the White Tower.

\subsection{Traffic Assignment and Simulation Models}

In the context of the present work macroscopic traffic assignment tools were used and their results were then introduced into microscopic traffic simulation tool. Fig. 2 shows the work flow that was followed.

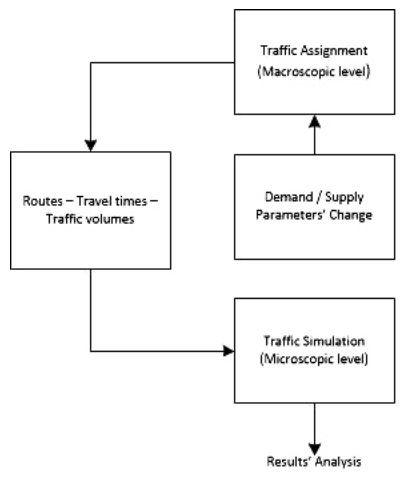

Fig. 2.

Workflow for Extraction and Analysis of the Results 


\section{1) Traffic Assignment Model}

The initial network, as coded in the traffic planning software VISUM used for various similar applications for the city of Thessaloniki, is composed of 290 nodes ( 91 of which are signalized) (Mitsakis et al., 2013) (Fig. 3). The connection of nodes is performed via 802 road sections that have geometrical (length, position in the network) and traffic characteristics (number of lanes, free flow speed, traffic direction, capacity of road section per lane, means of transport for which the use of the road section is allowed, existence of a bus-dedicated lane, use of the right/left lane for parking, number of public transport vehicles that pass per hour and per road section). 44 traffic analysis zones have been defined in the network and the travel demand is assigned to the road network via 104 connecting links (pseudo-road sections with marginal zero length), that connect the centroids of nodes with various nodes.

The travel demand of the network refers to the morning peak hour 08:00-09:00 and the origin-destination matrix has been corrected with traffic flow measurements that have been performed on a real time basis, at 17 spots of the network. The data are from the March of the year 2013 and the total number of movements is 19.632. The assignment of the network was made according to the Linear User Cost Equilibrium algorithm (Gentile and Noekel, 2009).

\section{2) Traffic Simulation Model}

The geometry of the network, as it has been coded in the traffic planning software, as well as the results of the traffic assignment process (routes, traffic volumes per trip and per road section, travel times, delays in the nodes) have been introduced in the traffic simulation model VISSIM (Fig. 3). The total number of road segments increased in 1.762 , as in this model the allowed turning movements are encoded as separate road sections. Afterwards, a traffic simulation was performed and it was followed by the analysis of the results.

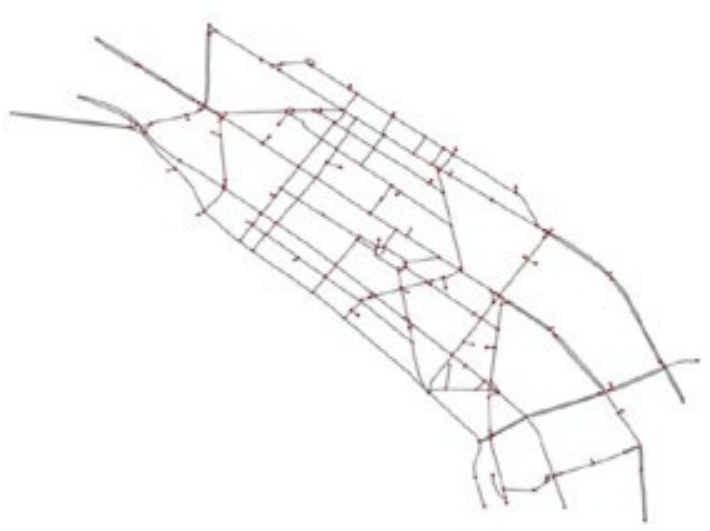

Fig. 3.

The Coded Network of Thessaloniki 


\subsection{Methodology of Results' Analysis}

For the development of the curve that correlates the traffic flow with density and the investigation of an MFD existence, the following process was followed:

- Traffic simulation in VISSIM;

- Storage of vehicles' flow and density information every 1 minute, for all the parts of the road network;

- Repetition of each simulation for five times with different random seeds;

- Performance of five hourly simulations.

Afterwards, for each evaluation time interval of the results, the following weighted averages were extracted, (0-1 hour for every 1 minute):

$$
q_{w}=\sum_{i} q_{i} \cdot l_{i} / \sum_{i} l_{i}
$$

$$
k_{w}=\sum_{i} k_{i} \cdot l_{i} / \sum_{i} l_{i}
$$

where $q_{w}$ is the weighted traffic volume, $q_{i}$ is the traffic volume at the road section $i, k_{w}$ is the weighted density, $k_{i}$ is the density at the road section $i$ and $l_{i}$ is the length of the road section $l_{i}$.

\subsection{Existence of a Flow-Density MFD}

The weighted averages of flow and density have been graphically correlated for all the random seeds. The results are depicted in Fig. 4, where the MFD for the central area of Thessaloniki is presented.

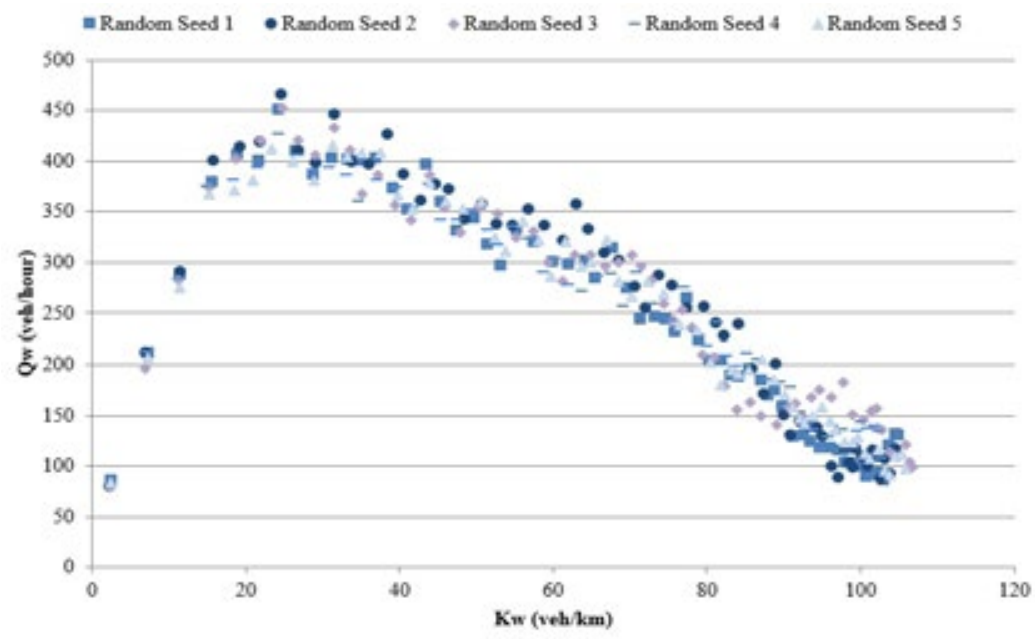

Fig. 4.

Macroscopic Fundamental Diagram Flow-Density for Thessaloniki's Road Network

As shown, the hourly weighted average capacity of the network for all the road sections is between 400 and 500 vehicles. The results are particularly concentrated, independently of each seed rate and do not exhibit significant dispersion. It is also apparent that the capacity point at which the network operates at the maximum value is in the range of 25 to 30 vehicles per kilometre. 


\section{Results' Analysis}

\subsection{Road Sections}

\subsubsection{Connecting Links}

In the framework of this analysis, the road sections that operate as connecting links and have the role to "carry" traffic in the network have been removed from the analysis. Therefore, the evaluation case of only realistic sections is examined, in which traffic and flow are more close to the reality. Road sections that operate as connecting links are totally 51. As shown in Fig. 5, the form and the MFD shape for both cases is the same, while the only difference observed is an immediate upwards displacement of the MFD for the connecting links, due to the additional volume that is transferred to the network through these road sections.

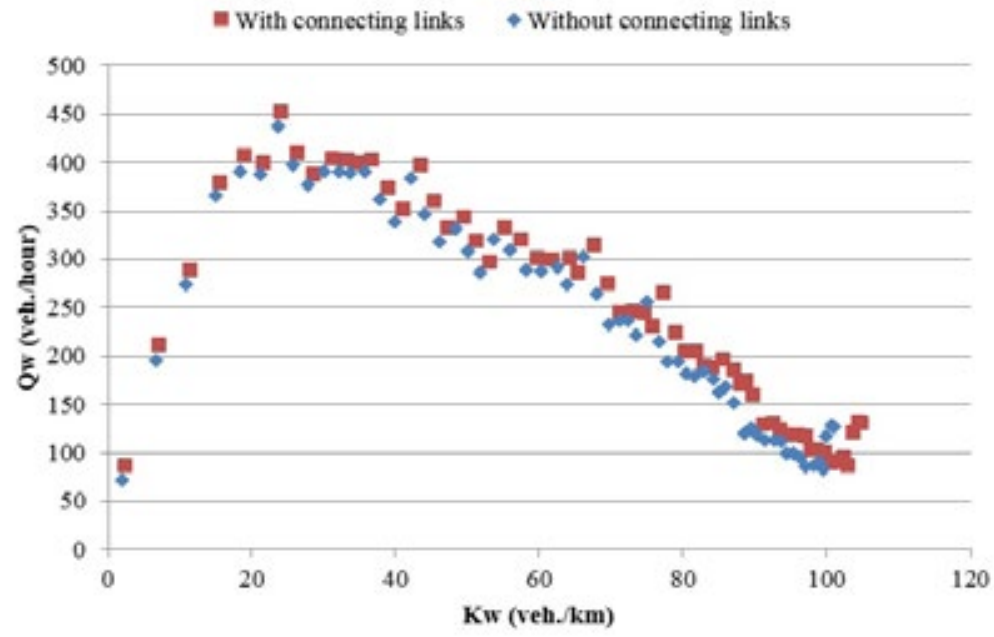

Fig. 5.

MFD Analysis with and without Connecting Links

\subsubsection{Clusters Analysis}

The initial analysis for the extraction of the flow-density MFD was performed in all the road sections of the network (1.762). In the present section, 10 different combinations of 880 ( 50\%), 440 ( 25\%), $210(\sim 12 \%)$, $110(\sim 6 \%), 60(\sim 3 \%)$ and $20(\sim 1 \%)$ road sections were examined and the minimum, average and maximum relative errors for the weighted average flow and density conditions were analysed. This analysis was made, so as the minimum number of road sections to be revealed, which evaluation is able to give representative results, as regards the MFD. The results are shown in Fig. 6 and in Fig. 7. 


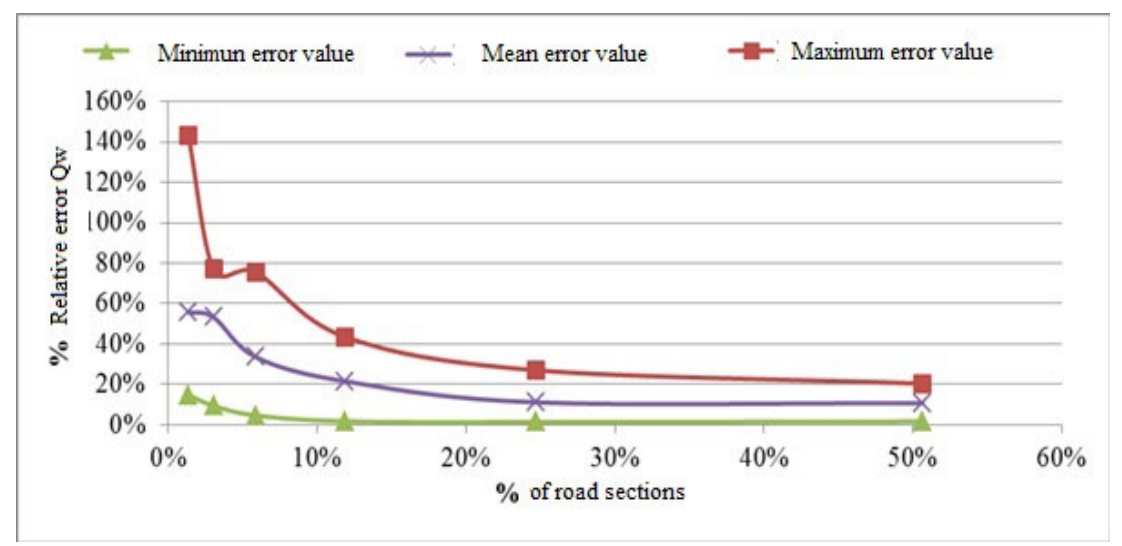

Fig. 6.

Percentage of Relevant Vehicle Flow Error per Total Number of Examined Road Sections

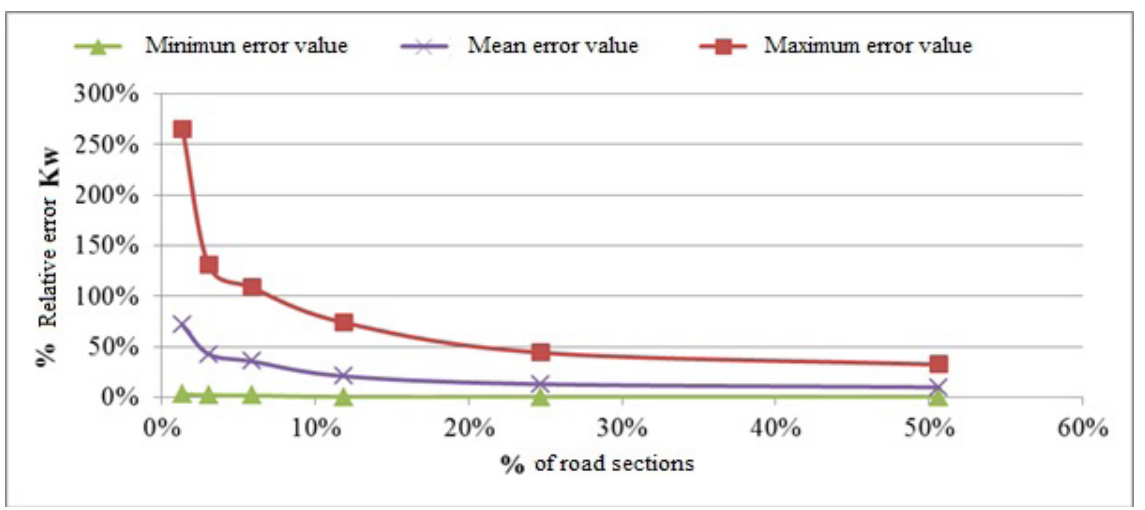

Fig. 7.

Percentage of Relevant Density Error per Total Number of Examined Road Sections

As it is presented in the above figures, both for the weighted average flow and the corresponding average density the relative error of the values is stabilized, compared to the initial condition, after the analysis of the $25 \%$ of all the road sections.

Therefore, this percentage can be considered as the basis for which the MFD may be representative of the network.

\subsection{Time Intervals-Duration Resolution}

\subsubsection{Time Intervals}

This section examines the variation in the shape and in the form of the MFD when the intervals' resolution (R.I. indicatively) is increased and decreased respectively. More specifically, except from the initial analysis of the one minute interval, intervals 
of two, five and ten minutes were used. The depicted in Fig. 8, along with their respective corresponding flow-density MFDs are fit curves.

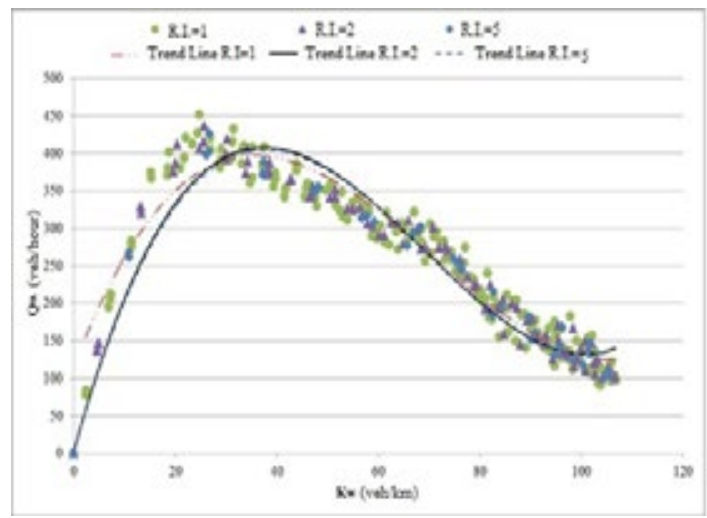

Fig. 8.

Macroscopic Fundamental Diagrams of Flow-Density for Different Time Intervals of Data Analysis

Fig. 8 shows that the time-interval resolution of one minute has a small dispersion, a fact that it is not revealed when the time-interval resolution is increased. The results of the analysis for the intervals of two and five minutes are similar. This shows that the two minutes-interval resolution is sufficient for an accurate network description and can be used in real-time applications.

\subsubsection{Duration of Resolution}

The network's evaluation lasts from minute one (1) up to minute sixtieth (60) when the 1 hour simulation is concluded. This section presents the results of a series of analyses, where the last time interval of the evaluation is extended for 30 minutes. The results are shown in Fig. 9.

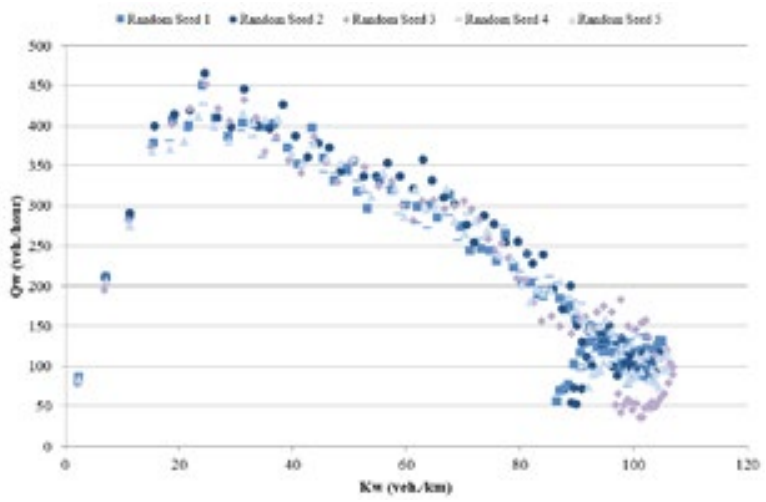

Fig. 9.

Macroscopic Fundamental Diagram of Flow-Density. Analysis Duration 0-4.800 Seconds 
The results show that a difference in the choice of the evaluation period duration is only observed in situations of high congestion in the network (density of 90100 vehicles per $\mathrm{km}$ ).

Nevertheless, in real conditions, at a network in which the travel demand is continuous (and it is not interrupted after one hour as in the traffic simulation), the flow-density MFD does not present the form that is shown in Fig. 9, at situations of high congestion.

\subsubsection{Travel Demand}

This section examines the sensitivity of the flow-density MFD of Thessaloniki's network, concerning changes in the total travel demand. As presented in Fig. 10, as the ratio of the total travel demand is over $+30 \%$ compared to the initial demand, then the form, shape and maximum/minimum values present a small dispersion, in contrast to the case when the ratio is below $-30 \%$ when the form and the shape of the MFD are the same, while the maximum/minimum values vary significantly.

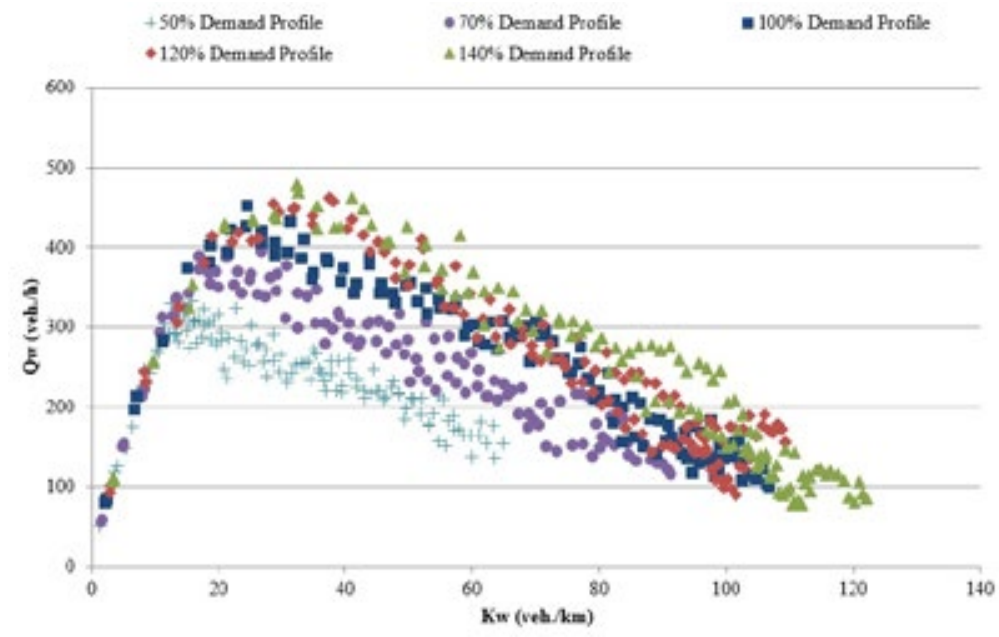

Fig. 10.

Macroscopic Fundamental Diagrams with Different Travel Demand Profiles

The difference in the maximum network capacity is attributed to the spatial distribution of the congestion, which is not homogeneous at lower demand levels.

\section{Conclusions}

According to the theory that has been presented and proved in previous papers (Daganzo and Geroliminis, 2008 ,
Geroliminis and Daganzo, 2007), MFDs are an approximate property of a network's structure that do not depend on the demand. Therefore, when they are estimated empirically, they can provide valuable information to operators so as to assess policies and measures related to traffic management aiming to improve mobility and exploit the maximum functionality of a network. 
Both the results presented in this paper and those of similar work in the literature are encouraging. They show that largescale networks behave predictively and independently of the specific origin-destination matrices. If these results are valid after further checks, then policy makers will have a reliable tool for predicting the effects of different traffic management policies and for future planning without the inherent uncertainty of current traffic forecasts and estimates. These further checks should include field measurements and further analyses, since simulations include various more or less realistic assumptions.

As derived from the results of the present work, areas of about $3 \mathrm{~km}^{2}$, as the one of Thessaloniki's city centre, have a clearly defined density-flow MFD. This MFD can be used as a tool for traffic control and accessibility improvement in the central area and on real-time basis, through the implementation from the perspective of public and private transport authorities, of pricing policies and of access control strategies, based on vehicles' accumulation. Such strategies have been used / or are currently used so far in Stockholm, London, Singapore and Mexico City. However, the possibility that is given through the MFD to monitor traffic situation continuously, can lead to the development of adaptive and customized traffic management policies for any area (e.g. the integration of this tool in the Urban Mobility Management system of Thessaloniki). Moreover MFD can be used for planning and assessing of new measures that are proposed for the city (e.g. pedestalization, new Public Transport).

The analysis of the results for the urban network of Thessaloniki shows that the correlation curve between the two variables is neither affected by the duration of the results' analysis nor by the frequency of the analysis intervals' resolution. It also appears to be only sensitive to the reduction of the total travel demand, while it preserves in parallel its form and shape (only the values of the average weighted flow and density are affected). Regarding the number of the road sections, which analysis can give representative results with respect to the density-flow MFD, it seems that at least an analysis of at least $25 \%$ of the total number of road sections is needed.

The next step concerning MFDs for Thessaloniki's road network should be towards the analysis of field measurements of various traffic data collection sources (inductive loops, cameras, Bluetooth sensors) in order to determine the MFD existence through empirical findings.

\section{References}

Buisson, Ch.; Ladier, C. 2009. Exploring the impact of the homogeneity of Traffic Measurement on the existence of macroscopic fundamental diagrams. In Proceedings of the 88th Annual Meeting of the Transportation Research Board, Transportation Research Board, U.S.A.

Courbon, T.; Leclercq, L. 2011. Cross-comparison of Macroscopic Fundamental Diagram Estimation Methods, Procedia Social and Behavioral Sceinecs. DOI: http://dx.doi.org/10.1016/j.sbspro.2011.08.048, 20: 417-426.

Cassidy, M.J.; Jang, K.; Daganzo, C. 2011. Macroscopic Fundamental Diagrams for Freeway Networks, Transportation Research Record, Transportation Research Board. DOI: http://dx.doi.org/10.3141/2260-02, 2660: 8-15.

Daganzo, C.F. 2005a. Improving city mobility through gridlock control: an approach and some ideas, Working Paper UCB-ITS-VWP-2005-1, U.C. Berkeley Center of Excellence on Future Urban Transport, University of California, Berkeley, CA. 
Daganzo, C.F. 2005b. A variational formulation of kinematic waves: Basic theory and complex boundary conditions, Transportation Research Part B: Methodological. DOI: http://dx.doi.org/10.1016/j.trb.2004.04.003, 39(2): 187-196.

Daganzo, C.F. 2007. Urban gridlock: macroscopic modeling and mitigation approaches, Transportation Research Part B: Methodological. DOI: http://dx.doi. org/10.1016/j.trb.2006.03.001, 41(1): 49-62.

Daganzo, C.F.; Geroliminis, N. 2008. An analytical approximation for the macroscopic fundamental diagram of urban traffic, Transportation Research Part B: Methodological. DOI: http://dx.doi.org/10.1016/j. trb.2008.06.008, 42(9): 771-781.

Gentile, G.; Noekel, K. 2009. Linear User Cost Equilibrium: the new algorithm for traffic assignment in VISUM. In Proceedings of European Transport Conference, Leeuwenhorst Conference Centre, Netherlands.

Geroliminis, N.; Daganzo, C.F. 2007. Macroscopic modeling of traffic in cities. Presented at the 86th Annual Meeting of the Transportation Research Board, paper \# 070413, Washington DC.

Geroliminis, N.; Sun, J. 2011a. Properties of a welldefined macroscopic fundamental diagram for urban traffic, Transportation Research Part B: Methodological. DOI: http://dx.doi.org/10.1016/j.trb.2010.11.004, 45(3): 605-617.

Geroliminis, N.; Sun, J. 2011b. Hysteresis phenomena of a Macroscopic Fundamental Diagram in freeway networks, Transportation Research Part A: Policy and Practice. DOI: http://dx.doi.org/10.1016/j.tra.2011.04.004, 45(9): 966-979.

Godfrey, J.W. 1969. The mechanism of a road network, Traffic Engineering \& Control, 8(8): 323-327.
Greenberg, H. 1959. An analysis of traffic flow, Operation Research, 7(1): 79-85.

Greenshields, B.D. 1934. A study of traffic capacity. In Proceedings of Highway Research Board, 14: 448-477.

Herman, R.; Ardekani, S.A. 1984. Characterizing traffic conditions in urban areas, Transportation Science. DOI: http://dx.doi.org/10.1287/trsc.18.2.101, 18(2): 101-140.

Herman, R.; Prigogine, I. 1979. A two-fluid approach to town traffic, Science, 204(4389): 148-151.

Ji, Y.; Geroliminis, N. 2011. Spatial and Temporal Analysis of Congestion in Urban Transportation Networks. In Proceedings of the 90th Annual Meeting of the Transportation Research Board, Transportation Research Board, U.S.A.

Knoop, V.L.; Hoogendoorn, S.P.; Van Lint, J.W.C. 2012. The impact of Traffic Dynamics on the Macroscopic Fundamental Diagram. In Proceedings of the 92nd Annual Meeting of the Transportation Research Board, Transportation Research Board, U.S.A.

Knoop, V.L.; Hoogendoorn, S.P. 2012. Empirics of a Generalized Macroscopic Fundamental Diagram. In Proceedings of the 92nd Annual Meeting of the Transportation Research Board, Transportation Research Board, U.S.A.

Laval, J.A. 2010. The Effect Of Signal Timing And Network Irregularities In The Macroscopic Fundamental Diagram. Presented at the Traffic Flow Theory Sumer Meeting, France.

Mahmassani, H.; Williams, J.C.; Herman, R. 1987. Performance of urban traffic networks. In: Gartner, N.H.; Wilson, N.H.M. (Eds.), 10th Int. Symp. on Transportation and Traffic Theory. Elsevier, Amsterdam, The Netherlands. 
Mazloumian, A.; Geroliminis, N.; Helbing, D. 2010. The Spatial Variability of Vehicle Densities 17 as Determinant of Urban Network Capacity, Philosophical Transactions of Royal Society A.

Mitsakis, E.; Stamos, I.; Grau, J.M.S.; Chrysochoou, E.; Iordanopoulos, P.; Aifadopoulou, G. 2013. Urban Mobility Indicators for Thessaloniki, Journal of Traffic and Logistics Engineering. DOI: http://dx.doi.org/10.12720/ jtle.1.2.148-152, 1(2): 148-152.

Stamos, I.; Kitis, G.; Basbas, S.; Tzevelekis, I. 2012. Evaluation of a High Occupancy Vehicle Lane in Central Business District Thessaloniki, Procedia-Social and Behavioral Sciences. DOI: http://dx.doi.org/10.1016/j. sbspro.2012.06.1085, 48: 1088-1096.

Stamos, I.; Kitis, G.; Basbas, S. 2013. The Implementation Of A Contra Flow Bus Lane In The City Of Thessaloniki: Energy And Environmental Impacts, Fresenius Environmental Bulletin, 22(7B): 2191-2196.
Politis, I.; Papaioannou, P.; Basbas, S.; Stamos, I. 2012. How Do Large-Scale Infrastructure Projects Contribute To Environmental Improvement. The Case Of Thessaloniki Outer Ring Road, Journal of Environmental Protection and Ecology, 13(1): 219-226.

Thomson, J.M. 1967. Speeds and flows of traffic in Central London, Traffic Engineering and Control, 8(12): 721-725.

Traffic Control Systems Handbook (TCSH). 2008. Control and Management Concepts for Freeways, US Department of Transportation, Federal Highway Administration.

Williams, J.C.; Mahmassani, H.S.; Herman, R. 1987. Urban Traffic Network Flow Models, Transportation Research Record, Transportation Research Board, 1112: 78-88.

Zahavi, Y. 1972. Traffic performance evaluation of road networks by the $\alpha$-relationship, Parts 1 and 2 - Traffic Engineering \& Control, 14(5 and 6): 228-231 and 292-293. 